\title{
Animal Models of Aging and Cognition
}

\author{
Joseph A. McQuail • Michelle M. Nicolle
}

Published online: 18 January 2012

(C) Springer Science+Business Media, LLC 2012

\begin{abstract}
Human aging is associated with variable rates of cognitive decline. Aged laboratory animals, specifically rats and monkeys, show similar cognitive profiles when tested on species-appropriate behavioral tasks. Using these cognitively defined animal models, we now understand that normal aging is associated with disconnections between functionally related brain regions that support specific behaviors. We review recent work describing molecular and cellular mechanisms that lead to decreased connections between the aged hippocampus and associated cortical and basal forebrain neurons required for normal memory. We also discuss behavioral evidence that aging leads to impaired executive functions with concurrent changes to key neural substrates within the frontostriatal network. Collectively, these studies demonstrate that cognitive deficits in older individuals are related to modest, circuit-specific alterations and that data obtained from such animal models can help to better inform the design of future clinical studies and improve neuropsychiatric outcomes for older patients.
\end{abstract}

Keywords Brain - Aging - Cognitive function - Cognitive aging $\cdot$ Cognition $\cdot$ Animal model $\cdot$ Memory $\cdot$ Spatial learning $\cdot$ Morris water maze $\cdot$ Executive function $\cdot$ Reversal learning - Delay discounting · Working memory .

J. A. McQuail

Program in Neuroscience, Wake Forest University,

1 Medical Center Boulevard,

Winston-Salem, NC 27157, USA

M. M. Nicolle $(\bowtie)$

Department of Internal Medicine, Section on Gerontology

and Department of Physiology \& Pharmacology,

Wake Forest University School of Medicine,

1 Medical Center Boulevard,

Winston-Salem, NC 27157, USA

e-mail: mnicolle@wakehealth.edu
Hippocampus · Medial temporal lobe - Entorhinal cortex · Basal forebrain $\cdot$ Prefrontal cortex $\cdot$ Striatum $\cdot$ Reelin . Acetylcholine $\cdot$ Calcium buffering $\cdot$ Dendritic spine . Afterhyperpolarization - Magnetic resonance imaging

\section{Introduction}

With improvements in the standard of living and medical innovation extending the human lifespan, more individuals will live to older ages. Consequently, the number of Americans over the age of 65 years will grow from about 40 million today to almost 90 million by 2050 , and will make up a larger fraction of our population, growing from $13 \%$ to $20 \%$ [1]. These demographic projections have important ramifications to the neuroscience research community because aging is associated with decreases in a number of cognitive domains, most notably memory [2]. However, age alone does not accurately predict cognitive ability, as older individuals exhibit a broad range of performance on neuropsychological tests. Some present with no or minimal decline; others demonstrate moderate, but selective, deficits; and the remainder display severe loss of function [3]. It is the interaction of chronological (or biological) aging with cognitive function that gives rise to the concept of "cognitive aging" (Fig. 1). Furthermore, subtle losses in cognitive function may predispose individuals to more severe outcomes. About $15 \%$ of those over the age of 70 years are diagnosed with mild cognitive impairment (MCI), presenting with memory deficits that are worse than expected even after correcting for age and level of education [4], and these patients are at increased risk for decline into dementia, most commonly Alzheimer's disease (AD) [5].

This article reviews the findings of several recent reports that examine alterations to neurobiological substrates of 


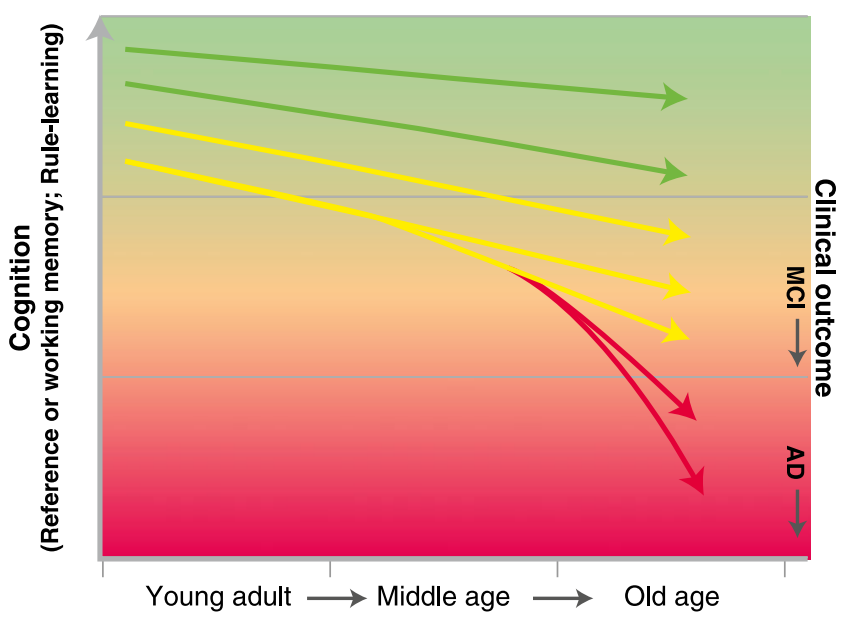

Fig. 1 The interaction between chronological or biological aging and cognitive function ("cognitive aging"). Cognitive function (shown on the left y-axis) generally declines with age (shown on the x-axis), but age alone is not sufficient to predict cognitive performance across all individuals of the same age because cognitive outcomes are more variable with advancing age. Some older individuals exhibit little or no cognitive impairment relative to younger individuals. Other individuals will present with modest, but significant, deficits in some cognitive domain, usually memory. Generally, these deficits are associated with selective changes to specific circuits. As cognitive deficits emerge in older individuals, there is increased risk for further decline into dementia, most frequently Alzheimer's disease, which is associated with neuropathology including neuron loss and brain atrophy. However, such diseases are distinct from normal aging, not an inevitable consequence. Even with improved clinical evaluations (right $y$-axis), the boundary that distinguishes moderate impairment from progressive neuropathology remains unclear (denoted by the color transitions of the background: green $=$ normal; orange $=$ mild impairment/at risk for dementia; red = probable dementia)

cognitive aging in animal models. We first focus on the medial temporal lobe network, discussing changes to cortical and basal forebrain neurons that innervate the hippocampus to support normal spatial learning. We then transition to a discussion of the frontostriatal system that supports behavioral flexibility and decision making. Finally, findings obtained from aged rodents will be extended by reviewing neurobiological assessments in aged monkeys obtained via traditional postmortem techniques, as well as in vivo brain imaging studies that bear closer resemblance to clinical approaches used in humans.

\section{Neurobiological Changes in Aged Rats with Spatial Learning Impairment}

\section{Assessing Reference Memory in Aged Rats}

Reference (or episodic) memory is largely dependent upon the integrity of the hippocampus and associated medial temporal lobe (MTL) structures, which are present in all mammalian species, indicating that rodents can be used to model the effects of aging on both cognition and brain substrates to address research questions of translational relevance to humans. The Morris water maze is a welldescribed apparatus for testing learning and memory in rats. The classical place-learning, or "hidden-platform," task is hippocampal-dependent, and aged rats (ie, 22-28 months of age, depending on the strain used) are impaired on this task relative to young controls (usually 4-6 months old) [6-9]. Importantly, when comparing performance of individual young and aged rats, there is greater variability in aged individuals; some aged rats exhibit performance similar to young rats while other aged rats are significantly worse than young rats [6]. This pattern of results closely approximates the cognitive aging phenotype conceptualized for aging humans (Fig. 1) and is apparent across the three strains of rats most commonly used in aging research: Long-Evans, Fischer 344 (F344), and F344 × Brown Norway (FBN) F1 Hybrids [10]. By using this translationally meaningful method to sort rats based upon behavioral criteria, rodent models of cognitive aging can more effectively identify changes to the aged brain that have a significant relationship to cognition, not merely age.

\section{Entorhinal Cortical Neurons and Memory Impairment}

The entorhinal cortex (EC) provides most cortical input to the hippocampus [11] and is an early locus of neuronal loss in patients with $\mathrm{AD}$ [12]. In a rodent model of cognitive aging, there is a loss of perforant path inputs to the hippocampus formed by layer II EC neurons specifically in aged rats with cognitive impairment [13]. The rodent pattern of denervation is similar to that observed in MCI patients [14] and provides an excellent model for identification of factors that render this projection vulnerable in aging. A current hypothesis stipulates that a loss of synaptic viability and plasticity leads to subsequent denervation. Reelin, a glycoprotein, is involved in synaptogenesis [15] and neuroplasticity [16] and is ideally positioned to influence perforant path connections because layer II EC neurons are among the few excitatory cell populations to express this protein [17]. Recently, Stranahan and colleagues [18••] reported that layer II of the EC of aged rats with cognitive impairment contains significantly fewer reelinergic neurons than young or aged rats with preserved spatial learning. In addition to hippocampal projections, these neurons form synapses within the EC and these connections are also lost in agedimpaired rats. Therefore, not only does the aged-impaired hippocampus receive less highly processed cortical input via the perforant path but remaining projections are likely less regulated as cortical synaptic integrity is compromised.

The work of Stranahan and colleagues $[18 \bullet \bullet, 19 \bullet \cdot]$ is an excellent example of a descriptive study that used an animal model of cognitive aging to inform further hypothesis- 
driven studies. Using insight from the cognitive aging mod$\mathrm{el}$, the importance of reelin to normal learning and memory was validated in a follow-up study by the same research group, demonstrating that intra-entorhinal infusion of recombinant receptor-associated protein (RAP), which blocks reelin from binding to its cognate receptor, produces significant spatial learning impairments in young rats [19••]. Furthermore, RAP infusion decreased phosphorylation of disabled-1, reelin's intracellular target, and decreased expression of the synaptic marker synaptophysin. Collectively, these studies demonstrate that reelin is a key molecular component of normal MTL cortical function, and its loss, either age-associated or experimentally induced, triggers profound synaptic loss and spatial-learning deficits.

\section{Cholinergic Basal Forebrain System and Memory Impairment}

Acetylcholine-producing neurons located in the basal forebrain (BF) give rise to fibers traveling via the fornix that terminate throughout the hippocampus [20]. Importantly, the release of acetylcholine controls switching between memory formation and retrieval processes in the hippocampus [21] and acetylcholine release is phase-locked with hippocampal theta oscillations [22], suggesting that normal cholinergic activity is closely linked to plasticity and learning. The loss of cholinergic cell bodies in the BF is well established in $\mathrm{AD}$ [23]. Initially, it was presumed that a similar loss was associated with normal aging; however, these early studies that reported a loss of BF cholinergic neurons in aging utilized profile-based counting methods [24-26]. Profile-based methods rely on a number of geometric assumptions that may confound comparisons between age groups. In contrast, stereological approaches make no such assumptions, but incorporate rigorous sampling methodologies to achieve reliable estimates of total cell number. Recently, stereological investigations have challenged the notion of BF cholinergic neuron loss in normal aging. Ypsilanti and colleagues [27•] reported that there was no change in total numbers of cholinergic neurons in the BF in 24-month-old versus 6-month-old F344 rats. Subsequently, McQuail and colleagues [28••] reported that this same cell population is also preserved in 28-month-old FBN rats with spatial learning impairment. Importantly, because stereological techniques were employed in both studies, it is significant that each reported strikingly similar numbers of cholinergic cells, thus validating both the same pattern of results as well as the total estimates between these two strains.

Although the stereological evidence indicates that $\mathrm{BF}$ cholinergic neurons do not degenerate with age, ongoing work suggests that other parameters of this cell population may contribute to cognitive aging. Ypsilanti and colleagues
[27•] reported reduced cholinergic fiber length in the aged hippocampus, suggesting a loss of hippocampal cholinergic synapses. Decreased connectivity between BF cholinergic neurons and hippocampus is reminiscent of lost perforant path connections, and similarly invites future investigations to identify the mechanisms that mediate this effect. Previously, it was reported that memory-impaired aged rats expressed fewer p75-containing neurons in the BF [29]. This observation is significant because p75 is a nerve growth factor receptor exclusive to cholinergic neurons within the BF, demonstrating selective loss of trophic support [30]. Although p75 expression was lowest in impaired aged rats, the relationship between the BF cholinergic system and spatial learning is complicated because selectively removing cholinergic fibers from the hippocampus does not induce spatial impairment in young rats [31,32].

The failure to recapitulate cognitive impairment via cholinergic lesion suggests that additional factors impact cholinergic function in the aged BF. Calcium dyshomeostasis forms a central theme in cognitive aging, especially considering that calcium regulates a variety of signaling cascades, modulates membrane excitability, and is necessary for synaptic plasticity [33]. With this in mind, calcium buffering is significantly increased in BF cholinergic neurons from aged learning-impaired F344 rats, whereas aged rats without impairment have lower calcium buffering values similar to young rats $[34 \cdot \bullet]$. Perhaps most interesting is that these changes in calcium buffering can be observed in middleaged rats (13 months) that do not yet exhibit behavioral impairments. This suggests that changes to calcium buffering within cholinergic neurons precede the manifestation of cognitive deficits, or that some middle-aged rats recruit adaptive mechanisms to regulate intracellular calcium and protect against subsequent cognitive decline. Collectively, these studies demonstrate that cholinergic degeneration is not a requisite feature of cognitive aging, but these neurons may form fewer connections with the hippocampus and exhibit selective changes in calcium buffering that may, in part, explain age-related cognitive deficits.

\section{Evaluating Age-Related Changes to Executive Function and Associated Brain Substrates in Aged Rats}

\section{Evaluating Executive Function in Aged Rats}

Executive function encompasses a number of cognitive processes dependent on the prefrontal cortex (PFC) that involve behavioral control, organization, and adaptation, including working memory, decision making, and behavioral flexibility. While not as widely studied in humans as memory deficits, neuropsychological testing has revealed executive function diminishes with increasing age $[2,35]$. 
Although not without debate, rodent models of executive function have been described $[36,37]$ and are being applied to the study of aging in rats $[38,39 \bullet \bullet, 40,41 \bullet \cdot$. Because there is no clear consensus on task parameters or specific components of executive function within the context of rodent cognitive aging, we will review the novel behavioral results reported in two studies examining reversal learning and decision making, respectively.

Reversal Learning and Muscarinic Function in Aged Rats

Nieves-Martinez and colleagues [39••] examined reversal learning in young (12 months), middle-aged (21 months), and aged (29 months) FBN rats using an attentional setshifting task where rats must dynamically monitor and modify their response strategy in light of changing task rules. In all stages, rats obtained food rewards hidden in pots scented with specific odorants (first dimension) and filled with distinct digging media (second dimension). In the first stage of this task, rats learned to discriminate between two odors (eg, lemon vs clove), where one (lemon) consistently identified the baited pot while the other (clove) was consistently unbaited. The digging medium is irrelevant and variable across testing. All rats regardless of age can reliably learn this rule; however, when the rule is reversed and the clove scent signaled food reward, aged rats required more trials than young or middle-aged rats to learn this rule reversal. In the last phase, the digging medium (eg, shredded paper or Easter grass), which was never previously associated with the food reward, became the salient signal for reward and odor was made irrelevant. Similar to the odor reversal, aged rats also were impaired at shifting between stimulus dimensions (ie, odor to digging medium); aged rats again required significantly more trials to reestablish performance criteria than young or middle-aged rats.

Following the completion of their behavioral studies, Nieves-Martinez and colleagues [39••] analyzed muscarinic receptors within the striatum, which interconnects with PFC, and found decreased receptor activity, without changes to receptor density, in the 24-month-old group. The timing and localization of these neurobiological changes suggest that deterioration in muscarinic striatal signaling is a factor in age-related reversal deficits. Other neurobiological alterations have been observed in frontostriatal circuitry in aged rats assessed in a similar manner, including changes in glutamate receptor binding [42]. Specifically, while both kainate and $N$ methyl-D-aspartate (NMDA)-receptor densities are decreased in the striatum of aged rats with impaired set-shifting ability, the relationship between receptor density and set shifting was unique for each subtype; higher kainite binding was associated with fewer errors while higher NMDA binding was associated with more errors. Given the divergent results observed for these two glutamate receptor subtypes as well as the functional alterations to muscarinic receptors, further studies are necessary to better elucidate the mechanisms that disrupt normal frontostriatal activity and associated behaviors, such as set shifting in aged rats.

\section{Decision Making in Aged Rats}

Temporal considerations often play a prominent role in decision making, particularly when one must choose between a very large reward that is not received until after a lengthy delay and an immediate smaller reward. From a behavioral economics perspective, the subjective value of the larger reward is diminished as the delay to receive the reward increases. This phenomenon is termed "delay discounting." While children and adolescents are more likely to select the immediate smaller reward than adults as the delay is increased [43], less is known with respect to how aged adults would behave in these tasks. Furthermore, it is not known if differences between younger and older adults are due to differences in subjective life experiences that may impact decision making or if aging modulates the neurological circuitry that controls decision making. The aged rat is an ideal model to test the latter hypothesis because it is possible to control for life experiences, even between age groups, to an extent that is not possible in humans. To assess the effects of age on delay discounting, Simon and colleagues $[41 \bullet \cdot$ ] presented young and aged F344 rats with a choice between a small (one pellet) but immediate food reward or a large (four pellets) food reward presented after a delay of up to $60 \mathrm{~s}$. Interestingly, aged rats did not "discount" the value of the larger food reward to the same extent as young rats even as the delay increased; aged rats consistently showed a preference to select the larger food reward up to the very longest delay, while younger rats began to favor smaller rewards even after the introduction of relatively brief delays (10-20 s). These rodent data suggest that aging likely modulates the neural circuitry necessary for decision making because rats, particularly those fed ad libitum within commercial and laboratory colonies, have minimal prior experience that would shape performance in such a task. However, Simon and colleagues [41 ••] did not analyze tissue from the rats used in this study, leaving interested readers to speculate as to the specific mechanisms that drive this shift in decision making in older rats.

\section{Changes in Cortical Function and Connectivity in Aged Monkeys with Working Memory Deficits}

Measuring Working Memory in Aged Monkeys

While investigations of frontal cortex have become more tractable in rodents with the development of more refined 
behavioral testing methodologies, nonhuman primates, specifically rhesus macaques, are translationally valuable to the study of cognitive aging owing to the greater homologies between human and monkey brains, especially with respect to cortical size and organization. The primate dorsolateral prefrontal cortex (dIPFC) is critical for normal working memory. Working memory in monkeys can be measured using the delayed response (DR) test, where monkeys must remember the location of a cue over the course of a brief delay before response selection. In contrast, recognition memory is often assessed in monkeys using a delayed non-matching to sample (DNMS) task. In this task, monkeys view a sample object, then, after a short delay, the monkey must select a novel object presented alongside the sample object to complete the trial and receive a food reward. While DR and DNMS may be respectively associated with working memory and recognition memory (and their affiliated neural circuits), this distinction is not complete because MTL lesions may impair DR performance [44] and the dIPFC is apparently necessary for DNMS acquisition $[45,46 \bullet$, $47 \cdot \bullet$. However, data obtained from these tasks consistently demonstrate working memory decline in monkeys starting after 20 years of age $[48,49]$. By using tissue from monkeys trained on these tasks, two recent publications have shed light on changes to the structure and physiology of dIPFC neurons in aged monkeys that may explain age-related impairments in working memory.

\section{Prefrontal Neurons and Working Memory Impairment}

While we have previously discussed evidence demonstrating that long projection pathways (perforant path and fornix) deteriorate with age, the effects of lost input on postsynaptic targets also warrant investigation. Dumitriu and colleagues [46••] offer evidence that working memory deficits are strongly associated with layer-specific changes to a particular subclass of dendritic spines. Spines are specialized postsynaptic structures that contain a high concentration of ionotropic glutamate receptors, and their morphology and receptor content are modulated by presynpatic activity. Specifically, smaller, thinner spines are more plastic and motile and express greater levels of NMDA receptors, while larger spines are more stable and express a greater proportion of AMPA ( $\alpha$ amino-3-hydroxy-5-methyl-4-isoxazolepropionic acid) receptors [50]. The existence of the more plastic "thin" spines is theorized to render the dIPFC more amenable to behavioral modification; conversely, larger spines have been stabilized by prior experience and resist alteration. Using a combination of traditional postmortem histological techniques, Dumitriu and colleagues [46••] observed that there was a significant age-related reduction in spine density within the dlPFC, but this reduction was specific to "thin" spines; the larger "mushroom" spines were not affected. Additionally, "thin" spines were, on average, larger in the aged dIPFC than those found in the young dlPFC. Most significantly, the lower density, as well as larger size, of "thin" spines in the aged dIPFC was highly correlated with poorer acquisition of the DNMS task. Thus, neurons in the aged-impaired dIPFC contain fewer modifiable spines and the loss of these most plastic substrates limits the efficacy of working memory processes.

Plasticity is dependent on not only spine morphology, but also neuronal activity. After normal learning, the afterhyperpolarization (AHP), a calcium-dependent current that brings the membrane potential to a hyperpolarized state after firing an action potential and modulates spiking frequency, is reduced to allow enhanced cell activity [51]. Traditional electrophysiological approaches have revealed that the AHP is increased in the hippocampus of aged rats with cognitive impairment [52], but similar data from PFC are scarce. To address this shortcoming, Luebke and Amatrudo [53・•] performed in vitro recording experiments using tissue from young and aged monkeys to determine whether the AHP and other electrophysiological parameters of neurons in the dIPFC are changed by age and associated with working memory. Importantly, parameters of layer III neurons, which connect to other cortical regions, were contrasted with layer $\mathrm{V}$ neurons, which connect primarily to subcortical regions. Using this strategy, Luebke and Amatrudo [53••] found that the AHP was significantly increased in layer III neurons, and greater increases in the AHP current were associated with worse working memory performance. While an increase in AHP was initially hypothesized to decrease cell firing, the firing rate of layer III neurons was actually increased in aged monkeys and not related to AHP. These divergent results suggest that some other factor, such as intracellular calcium (which can regulate both cell excitability as well as AHP amplitude), may influence these parameters. Interestingly, these robust changes apparent in layer III neurons of aged monkeys were not present in layer $\mathrm{V}$; this layer-specific effect may indicate that circuit-level changes in the aged PFC play some role in the manifestation of enhanced AHP.

The morphological data of Dumitriu and colleagues [46••] demonstrate that aged dIPFC is less amenable to modification by "thin" spine loss, and Luebke and Amatrudo [53••] present congruent physiologic evidence that dIPFC neurons exhibit enhanced AHP, rendering these neurons less adaptive to subsequent activity-dependent modulation. The significance of these alterations to normal cognition was supported in each study by reliable associations between age-related changes to "thin" spine density or AHP and severity of working memory deficits.

Assessing Brain Systems and Working Memory Impairment

The anatomical similarities between the human and monkey brain not only facilitate translational research through ex 
vivo morphological and physiological assessments, but also permit the use of brain imaging technology identical to that employed in clinical settings for human patients. Human studies of aging generally require large numbers of participants to achieve reliable results separating true effects of aging from confounds, including health factors, lifestyle, and other experiences that can affect clinical outcomes. It is possible to control for many of these factors in aged monkeys and, therefore, conduct well-powered imaging studies using fewer subjects while yielding results that can directly inform human aging studies and clinical practice. Perhaps of greatest interest, the use of noninvasive in vivo brain imaging techniques opens the door to future longitudinal studies of brain structure and behavior that could not be accomplished using the ex vivo approaches that have traditionally defined neurological assessments of the aged brain.

Using structural magnetic resonance imaging (MRI), Shamy and colleagues [47••] compared regional volumes between young and aged monkeys and determined the dlPFC and striatum both are smaller in aged monkeys. Furthermore, smaller volumes in both of these regions were significantly associated with poorer working memory. This study elegantly demonstrates that normal aging is not associated with widespread cerebral atrophy; rather, specific brain regions are more sensitive to the aging process. Interestingly, the brain regions that were identified in these aged monkeys, PFC and striatum, comprise the frontostriatal network that is also compromised in aged rodents [39••, 42]. However, disruptions to the integrity of neural networks are related to changes not only in grey matter, but also the white matter (WM) fiber tracts that contain axons that convey information between brain regions. Shamy and colleagues $[47 \bullet \cdot$ ] revealed that a tendency toward decreased frontal WM volume in older monkeys was associated with poorer DNMS performance. Similarly, Wisco and colleagues [54•] reported decreased frontal WM volume in aged monkeys, although this reduction was not related to cognitive impairment. Despite general agreement, core differences in the conclusions of these studies warrant a more refined analysis of WM integrity in the aged PFC. Accordingly, Makris and colleagues [55] utilized diffusion tensor MRI (DT-MRI), which measures the directional diffusion of water along myelinated axons, to evaluate the integrity of discrete fiber pathways that connect with the PFC. It was found that the integrity of the superior longitudinal fasciculus, the cingulum bundle, and anterior corpus callosum were compromised in older monkeys. This DT-MRI evidence was complimented by a subsequent histological analysis that reported both a decrease in myelinated axon density and increased frequency of myelin degeneration in the cingulum and anterior corpus callosum of aged monkeys [56••]. Because each of these fiber pathways serves to interconnect the
PFC across hemispheres and to other cortical regions, it is notable that both studies found that indices of myelin damage, either DT-MRI or histologically-derived, were associated with impairments in working memory $[55,56 \bullet \bullet$.

\section{Conclusions}

Even in the absence of any overt neurological illness, aging is associated with a decline in cognitive capacities. The severity of this decline is highly variable, with some individuals showing little evidence of loss and others presenting with more obvious deficits. Despite species differences, many of the key neural circuits are present in traditional laboratory animals, including rodents and monkeys, and species-appropriate tasks have revealed age-related declines in cognitive function similar to humans. Using these cognitively defined models, we now appreciate that normal aging is free from widespread neurodegeneration. However, we do observe a selective loss of synapses and connections and changes to neuron physiology that compromise the functions of brain networks that support normal cognition. These subtle changes may, in turn, predispose the aged brain to the subsequent manifestation of pathological conditions such as AD. However, in the absence of pathology, the more modest changes observed in nonpathological aging also may provide a window of opportunity to intervene and prevent cognitive decline. This latter consideration underscores the need for future work to develop novel therapies that better apply this knowledge regarding the mechanistic basis of cognitive aging.

Disclosures No potential conflicts of interest relevant to this article were reported.

\section{References}

Papers of particular interest, published recently, have been highlighted as:

- Of importance,

•- Of major importance

1. United States Census Bureau: 2009 U.S. Population Projections. Available at: http://www.census.gov/population/www/projections/ 2009projections.html. Accessed November 2011.

2. Salthouse TA. Are individual differences in rates of aging greater at older ages? Neurobiol Aging. 2011; In press.

3. Christensen H, Mackinnon AJ, Korten AE, et al. An analysis of diversity in the cognitive performance of elderly community dwellers: individual differences in change scores as a function of age. Psychol Aging. 1999;14:365-79. 
4. Roberts RO, Geda YE, Knopman DS, et al. The mayo clinic study of aging: design and sampling, participation, baseline measures and sample characteristics. Neuroepidemiology. 2008;30:58-69.

5. Petersen RC, Roberts RO, Knopman DS, et al. Mild cognitive impairment: ten years later. Arch Neurol. 2009;66:1447-55.

6. Gallagher M, Burwell R, Burchinal M. Severity of spatial learning impairment in aging: development of a learning index for performance in the Morris water maze. Behav Neurosci. 1993;107:618-26.

7. Frick KM, Baxter MG, Markowska AL, et al. Age-related spatial reference and working memory deficits assessed in the water maze. Neurobiol Aging. 1995;16:149-60.

8. Wyss JM, Chambless BD, Kadish I, et al. Age-related decline in water maze learning and memory in rats: strain differences. Neurobiol Aging. 2000;21:671-81

9. Markowska AL, Savonenko A. Retardation of cognitive aging by life-long diet restriction: implications for genetic variance. Neurobiol Aging. 2002;23:75-86.

10. LaSarge CL, Nicolle MM. Comparison of different cognitive rat models of human aging. In: Bizon JL, Woods AG, editors. Animal models of human cognitive aging. New York: Humana Press; 2009. p. 73-102.

11. van Strien NM, Cappaert NL, Witter MP. The anatomy of memory: an interactive overview of the parahippocampal-hippocampal network. Nat Rev Neurosci. 2009;10:272-82.

12. Blennow K, de Leon MJ, Zetterberg H. Alzheimer's disease. Lancet. 2006;368:387-403.

13. Smith TD, Adams MM, Gallagher M, et al. Circuit-specific alterations in hippocampal synaptophysin immunoreactivity predict spatial learning impairment in aged rats. J Neurosci. 2000;20:6587-93.

14. Scheff SW, Price DA, Schmitt FA, et al. Hippocampal synaptic loss in early Alzheimer's disease and mild cognitive impairment. Neurobiol Aging. 2006;27:1372-84.

15. Borrell V, Del Rio JA, Alcantara S, et al. Reelin regulates the development and synaptogenesis of the layer-specific entorhinohippocampal connections. J Neurosci. 1999;19:1345-58.

16. Chin J, Massaro CM, Palop JJ, et al. Reelin depletion in the entorhinal cortex of human amyloid precursor protein transgenic mice and humans with Alzheimer's disease. J Neurosci. 2007;27:2727-33.

17. Ramos-Moreno T, Galazo MJ, Porrero C, et al. Extracellular matrix molecules and synaptic plasticity: immunomapping of intracellular and secreted reelin in the adult rat brain. Eur J Neurosci. 2006;23:401-22.

18. • Stranahan AM, Haberman RP, Gallagher M. Cognitive decline is associated with reduced reelin expression in the entorhinal cortex of aged rats. Cereb Cortex. 2011;21:392-4. This article reports a loss of reelinergic entorhinal neurons specific to aged rats with cognitive impairment. Given prior work describing lost perforant path connections arising from the entorhinal cortex in agedimpaired rats, the authors argue that reelin may be a central molecule in mediating this effect.

19. • Stranahan AM, Salas-Vega S, Jiam NT, et al. Interference with reelin signaling in the lateral entorhinal cortex impairs spatial memory. Neurobiol Learn Mem. 2011; 96:150-5. Having previously observed decreased reelin in the entorhinal cortex of aged rats with spatial learning impairment, the authors of this paper demonstrate that experimentally blocking reelin activity in the entorhinal cortex of young rats will induce age-like spatial learning deficits. Similarly, decreased reelin activity also triggers synapse loss within the entorhinal cortex.

20. Amaral DG, Kurz J. An analysis of the origins of the cholinergic and noncholinergic septal projections to the hippocampal formation of the rat. J Comp Neurol. 1985;240:37-59.

21. Rogers JL, Kesner RP. Cholinergic modulation of the hippocampus during encoding and retrieval. Neurobiol Learn Mem. 2003;80:332-42.
22. Zhang H, Lin SC, Nicolelis MA. Spatiotemporal coupling between hippocampal acetylcholine release and theta oscillations in vivo. J Neurosci. 2010;30:13431-40.

23. Whitehouse PJ, Price DL, Clark AW, et al. Alzheimer disease: evidence for selective loss of cholinergic neurons in the nucleus basalis. Ann Neurol. 1981;10:122-6.

24. Armstrong DM, Sheffield R, Buzsaki G, et al. Morphologic alterations of choline acetyltransferase-positive neurons in the basal forebrain of aged behaviorally characterized Fisher 344 rats. Neurobiol Aging. 1993;14:457-70.

25. Baskerville KA, Kent C, Nicolle MM, et al. Aging causes partial loss of basal forebrain but no loss of pontine reticular cholinergic neurons. Neuroreport. 2006;17:1819-23.

26. Fischer W, Chen KS, Gage FH, et al. Progressive decline in spatial learning and integrity of forebrain cholinergic neurons in rats during aging. Neurobiol Aging. 1992;13:9-23.

27. - Ypsilanti AR, Girao da Cruz MT, Burgess A, et al. The length of hippocampal cholinergic fibers is reduced in the aging brain. Neurobiol Aging. 2008; 29:1666-79. This paper used rigorous stereological methodology to reveal that cholinergic neurons are not lost in the basal forebrain of aged rats. However, in these same aged rats, there is a significant reduction in cholinergic fiber length within the hippocampus. The authors argue that reduced innervation of hippocampus by cholinergic projections may explain cognitive impairments, although behavioral testing was not a component of this investigation.

28. •• McQuail JA, Riddle DR, Nicolle MM. Neuroinflammation not associated with cholinergic degeneration in aged-impaired brain. Neurobiol Aging. 2011; 32:2322 e1-4. This paper used stereological approaches to determine that basal forebrain cholinergic neurons are not lost in rats with behaviorally confirmed spatial learning impairments. This paper conclusively demonstrated that cholinergic degeneration is not necessary to observe cognitive impairment in aged rats.

29. Greferath U, Bennie A, Kourakis A, et al. Impaired spatial learning in aged rats is associated with loss of p75-positive neurons in the basal forebrain. Neuroscience. 2000;100:363-73.

30. Cuello AC, Bruno MA, Bell KF. NGF-cholinergic dependency in brain aging, MCI and Alzheimer's disease. Curr Alzheimer Res. 2007;4:351-8.

31. Chappell J, McMahan R, Chiba A, et al. A re-examination of the role of basal forebrain cholinergic neurons in spatial working memory. Neuropharmacology. 1998;37:481-7.

32. Fletcher BR, Baxter MG, Guzowski JF, et al. Selective cholinergic depletion of the hippocampus spares both behaviorally induced Arc transcription and spatial learning and memory. Hippocampus. 2007; 17:227-34

33. Foster TC. Calcium homeostasis and modulation of synaptic plasticity in the aged brain. Aging Cell. 2007;6:319-25.

34. •• Murchison D, McDermott AN, Lasarge CL, et al. Enhanced calcium buffering in F344 rat cholinergic basal forebrain neurons is associated with age-related cognitive impairment. J Neurophysiol. 2009; 102:2194-207. This paper used electrophysiological techniques to determine that calcium buffering is enhanced in basal forebrain cholinergic neurons of aged-impaired rats, while aged-unimpaired rats had buffering capacity similar to young. Furthermore, the calcium-buffering enhancement was observed in middle-aged rats that do not exhibit spatial learning deficits, suggesting that alterations to calcium buffering precede overt cognitive impairment.

35. Salthouse TA. What cognitive abilities are involved in trail-making performance? Intelligence. 2011;39:222-32.

36. Chudasama Y. Animal models of prefrontal-executive function. Behav Neurosci. 2011;125:327-43.

37. Kesner RP, Churchwell JC. An analysis of rat prefrontal cortex in mediating executive function. Neurobiol Learn Mem. 2011;96:417-31. 
38. Barense MD, Fox MT, Baxter MG. Aged rats are impaired on an attentional set-shifting task sensitive to medial frontal cortex damage in young rats. Learn Mem. 2002;9:191-201.

39. • Nieves-Martinez E, Haynes K, Childers SR, et al. Muscarinic receptor/G-protein coupling is reduced in the dorsomedial striatum of cognitively impaired aged rats. Behav Brain Res. 2011. This paper examined cognitive flexibility, a core component of executive function, in aged rats and observed age-related impairments in reversal-learning and set shifting. The emergence of these deficits was accompanied by decreased muscarinic receptor activity within the aged striatum.

40. Schoenbaum G, Nugent S, Saddoris MP, et al. Teaching old rats new tricks: age-related impairments in olfactory reversal learning. Neurobiol Aging. 2002;23:555-64.

41. •- Simon NW, LaSarge CL, Montgomery KS, et al. Good things come to those who wait: attenuated discounting of delayed rewards in aged Fischer 344 rats. Neurobiol Aging. 2011; 31:853-62. This paper investigated age-related changes in decision making, another important aspect of executive function. The observations strongly suggest that aging, separate from subjective life experiences, modulates decision making, possibly in a circuit-dependent fashion.

42. Nicolle MM, Baxter MG. Glutamate receptor binding in the frontal cortex and dorsal striatum of aged rats with impaired attentional set-shifting. Eur J Neurosci. 2003;18:3335-42.

43. Green L, Myerson J, Lichtman D, et al. Temporal discounting in choice between delayed rewards: the role of age and income. Psychol Aging. 1996;11:79-84.

44. Zola-Morgan S, Squire LR, Amaral DG. Human amnesia and the medial temporal region: enduring memory impairment following a bilateral lesion limited to field CA1 of the hippocampus. J Neurosci. 1986;6:2950-67.

45. Peters A, Sethares C, Luebke JI. Synapses are lost during aging in the primate prefrontal cortex. Neuroscience. 2008;152:970-81.

46. •• Dumitriu D, Hao J, Hara Y, et al. Selective changes in thin spine density and morphology in monkey prefrontal cortex correlate with aging-related cognitive impairment. J Neurosci. 2011; 30:750715. This paper reported a selective loss of thin spines in the prefrontal cortex of aged monkeys with working memory impairment. In contrast, mushroom spines, which resist alteration, are not lost with age. The authors argue that the loss of thin spines is critical for executive function because these spines are highly plastic and likely support novel rule-learning.

47. •- Shamy JL, Habeck C, Hof PR, et al. Volumetric correlates of spatiotemporal working and recognition memory impairment in aged rhesus monkeys. Cereb Cortex. 2011; 21:1559-73. This paper used MRI to measure regional volumes throughout the entire primate brain. They found selective reductions in prefrontal and striatal volumes that correlated with working memory performance.

48. Herndon JG, Moss MB, Rosene DL, et al. Patterns of cognitive decline in aged rhesus monkeys. Behav Brain Res. 1997;87:25-34.

49. Moore TL, Killiany RJ, Herndon JG, et al. Executive system dysfunction occurs as early as middle-age in the rhesus monkey. Neurobiol Aging. 2006;27:1484-93.

50. Bourne J, Harris KM. Do thin spines learn to be mushroom spines that remember? Curr Opin Neurobiol. 2007;17:381-6.

51. Matthews EA, Linardakis JM, Disterhoft JF. The fast and slow afterhyperpolarizations are differentially modulated in hippocampal neurons by aging and learning. J Neurosci. 2009;29:4750-5.

52. Tombaugh GC, Rowe WB, Rose GM. The slow afterhyperpolarization in hippocampal CA1 neurons covaries with spatial learning ability in aged Fisher 344 rats. J Neurosci. 2005;25:2609-16.

53. • Luebke JI, Amatrudo JM. Age-related increase of sI(AHP) in prefrontal pyramidal cells of monkeys: relationship to cognition. Neurobiol Aging. 2011. This paper reported increased AHP amplitude in layer III neurons of aged monkeys is associated with worse working memory performance. Increased AHP was not related to cell firing, which also was increased in aged monkeys. These data reveal layer-specific changes in prefrontal neuron activity, although the basis for the changes warrants additional investigation.

54. - Wisco JJ, Killiany RJ, Guttmann CR, et al. An MRI study of agerelated white and gray matter volume changes in the rhesus monkey. Neurobiol Aging. 2008; 29:1563-75. This paper used structural MRI to reveal that prefrontal grey and white matter volumes are reduced in aged monkeys. However, these reductions were not specifically associated with working memory impairment.

55. Makris N, Papadimitriou GM, van der Kouwe A, et al. Frontal connections and cognitive changes in normal aging rhesus monkeys: a DTI study. Neurobiol Aging. 2007;28:1556-67.

56. •• Bowley MP, Cabral H, Rosene DL, et al. Age changes in myelinated nerve fibers of the cingulate bundle and corpus callosum in the rhesus monkey. J Comp Neurol. 2010; 518:3046-64. This paper used electron microscopy to directly evaluate changes to myelinated fibers in monkey frontal white matter pathways. In aged monkeys, the density of myelinated fibers was decreased while signs of myelin degeneration were increased. This paper provides useful histological evidence to support DTI findings. 